\title{
Numerical Solution of Nonlinear Sine-Gordon Equation by Modified Cubic B-Spline Collocation Method
}

\author{
R. C. Mittal and Rachna Bhatia \\ Department of Mathematics, IIT Roorkee, Roorkee, Uttarakhand 247667, India \\ Correspondence should be addressed to Rachna Bhatia; rachnabhsingh@yahoo.com
}

Received 11 May 2014; Accepted 16 July 2014; Published 10 August 2014

Academic Editor: Nikolai A. Kudryashov

Copyright (C) 2014 R. C. Mittal and R. Bhatia. This is an open access article distributed under the Creative Commons Attribution License, which permits unrestricted use, distribution, and reproduction in any medium, provided the original work is properly cited.

Modified cubic B-spline collocation method is discussed for the numerical solution of one-dimensional nonlinear sine-Gordon equation. The method is based on collocation of modified cubic B-splines over finite elements, so we have continuity of the dependent variable and its first two derivatives throughout the solution range. The given equation is decomposed into a system of equations and modified cubic B-spline basis functions have been used for spatial variable and its derivatives, which gives results in amenable system of ordinary differential equations. The resulting system of equation has subsequently been solved by SSP-RK54 scheme. The efficacy of the proposed approach has been confirmed with numerical experiments, which shows that the results obtained are acceptable and are in good agreement with earlier studies.

\section{Introduction}

In this paper we consider the one-dimensional sine-Gordon equation

$$
\frac{\partial^{2} u}{\partial t^{2}}=\frac{\partial^{2} u}{\partial x^{2}}-\sin (u), \quad x \in\left(L_{1}, L_{2}\right), t \geq 0
$$

with initial conditions

$$
u(x, 0)=\phi_{1}(x), \quad u_{t}(x, 0)=\phi_{2}(x) .
$$

The Dirichlet boundary conditions are given by

$$
u\left(L_{1}, t\right)=\psi_{1}(t), \quad u\left(L_{2}, t\right)=\psi_{2}(t), \quad t \geq 0 .
$$

The nonlinear sine-Gordon equation arises in many different applications such as propagation of fluxion in Josephson junctions [1], differential geometry, stability of fluid motion, nonlinear physics, and applied sciences [2]. The sine-Gordon equation (1) is a particular case of Klein-Gordon equation, which plays a significant role in many scientific applications such as solid state physics, nonlinear optics and quantum field theory [3], given by

$$
\frac{\partial^{2} u}{\partial t^{2}}-\alpha \frac{\partial^{2} u}{\partial x^{2}}+g(u)=f(x, t),
$$

where $g(u)$ is a nonlinear force and $\alpha$ is a constant.

In the literature several schemes have been developed for the numerical solution of sine-Gordon equation. Ben-Yu et al. [4] proposed two difference schemes; Bratsos and Twizell [5] used method of lines to transform the initial/boundary value problem associated with (1) into a first order nonlinear initial value problem. Mohebbi and Dehghan [6] presented a combination of a compact finite difference approximation of fourth order and a fourth-order A-stable DIRKN method. Kuang and $\mathrm{Lu}$ [7] proposed two classes of finite difference method for generalized sine-Gordon equation; Bratsos and Twizell [8] presented a family of finite difference method, in which time and space derivatives are replaced by finite-difference approximations and then the equation is converted into a linear algebraic system. Wei [9] used the discrete singular convolution algorithm for the integration of (1). A variational 
TABLE 1: The values of $B_{j}(x)$ and its derivatives.

\begin{tabular}{lccccc}
\hline$x$ & $x_{j-2}$ & $x_{j-1}$ & $x_{j}$ & $x_{j+1}$ & $x_{j+2}$ \\
\hline$B_{j}(x)$ & 0 & 1 & 4 & 1 & 0 \\
$B_{j}^{\prime}(x)$ & 0 & $\frac{3}{h}$ & 0 & $-\frac{3}{h}$ & 0 \\
$B_{j}^{\prime \prime}(x)$ & 0 & $\frac{6}{h^{2}}$ & $-\frac{12}{h^{2}}$ & $\frac{6}{h^{2}}$ & 0 \\
\hline
\end{tabular}

iteration method to obtain approximate analytical solution of the sine-Gordon equation without any discretization has been developed by Batiha et al. [10]. Zheng [11] presented a numerical solution of sine-Gordon equation defined on the whole real axis. Bratsos [12] used a fourth-order rational approximation to the matrix exponential term in a threetime level recurrence relation for the numerical solution of (1). Dehghan and Shokri [13] solved the equation using collocation points and approximate the solution using radial basis functions; Dehghan and Mirzaei [14] used a boundary integral equation method; Rashidinia and Mohammadi [15] developed two implicit finite difference schemes, by using spline function approximations. Li-Min and Zong-Min [16] presented a meshless scheme by using a multiquadric quasiinterpolation without solving a large-scale linear system of equations, but a polynomial was needed to improve the accuracy of the scheme, while Jiang and Wang [17] proposed meshless approach by directly using high accuracy MQ quasiinterpolation without using any polynomial. A modified decomposition method for explicit and numerical solutions of the sine-Gordon equation in the form of convergent power series has been proposed by Kaya [18]. Uddin et al. [19] proposed a meshfree approach based on radial basis function for numerical solution of (1).

The B-spline possesses several properties such as minimal compact support and smoothness, which makes them suitable for the numerical solution of linear and nonlinear partial differential equations. B-spline with collocation provides a very simple solution procedure of differential equations. They also produce a spline function which is useful to obtain the solution at any point of the domain, while in finite difference methods $[4,6-8,15]$, we can find the solution only at the selected knots. In the present method, approximate solutions of sine-Gordon equation are obtained using a modified cubic $\mathrm{B}$-spline collocation method (MCBCM) in space and strong stability preserving Runge-Kutta (SSP-RK54) scheme [20] in time. The equation is converted into a system of partial differential equations and then, using $\mathrm{MCBCM}$, it reduces into a system of ordinary differential equations. Finally we use SSP-RK54 scheme to solve the obtained system of ODEs. Numerical solution of nonlinear sine-Gordon equation has been obtained without using any transformation or without linearizing the nonlinear term.

The paper is organized as follows. In Section 2, cubic Bspline collocation method is explained. In Section 3, modified cubic B-spline basis functions are introduced and how to find the solution of (1)-(3) using these basis functions is explained. Initial vectors have been computed in Section 4. Numerical experiments are conducted in Section 5, to demonstrate the viability and the efficiency of the proposed method computationally, and results are compared with some previous results. Finally, brief conclusions drawn from the present study are presented in Section 6.

\section{Description of Method}

The solution domain $L_{1} \leq x \leq L_{2}$ is partitioned into a mesh of uniform length $h=x_{j+1}-x_{j}$, where $j=0,1,2, \ldots, N-1, N$, such that $L_{1}=x_{0}<x_{1}<\cdots<x_{N-1}<x_{N}=L_{2}$.

In the cubic $\mathrm{B}$-spline collocation method the approximate solution can be written as the linear combination of cubic B-spline basis functions for the approximation space under consideration. Our numerical treatment for solving (1) using the collocation method with cubic B-Spline is to find an approximate solution $U_{N}(x, t)$ to the exact solution $u(x, t)$ in the form

$$
U_{N}(x, t)=\sum_{j=-1}^{N+1} c_{j}(t) B_{j}(x),
$$

where $c_{j}(t)$ are the time dependent quantities to be determined from boundary conditions and collocation from the differential equation.

The cubic B-spline $B_{j}(x)$ at the knots is given by

$$
\begin{aligned}
& B_{j}(x) \\
& \quad=\frac{1}{h^{3}} \begin{cases}\left(x-x_{j-2}\right)^{3} & x \in\left[x_{j-2}, x_{j-1}\right), \\
\left(x-x_{j-2}\right)^{3}-4\left(x-x_{j-1}\right)^{3} & x \in\left[x_{j-1}, x_{j}\right), \\
\left(x_{j+2}-x\right)^{3}-4\left(x_{j+1}-x\right)^{3} & x \in\left[x_{j}, x_{j+1}\right), \\
\left(x_{j+2}-x\right)^{3} & x \in\left[x_{j+1}, x_{j+2}\right), \\
0 & \text { otherwise, }\end{cases}
\end{aligned}
$$

where the set of functions $\left\{B_{-1}, B_{0}, B_{1}, \ldots, B_{N-1}, B_{N}, B_{N+1}\right\}$ forms a basis for the function defined over the region $L_{1} \leq$ $x \leq L_{2}$ with the obvious adjustment of the boundary base functions to avoid undefined knots. Each cubic B-spline covers four elements so that an element is covered by four cubic B-splines. The values of $B_{j}(x)$ and its derivatives are tabulated in Table 1.

Then, using approximate function (5) and Table 1, the approximate values of $U_{N}(x, t)$ and its two derivatives at the knots are determined in terms of the time parameters $c_{j}$ as follows:

$$
\begin{gathered}
U_{j}=c_{j-1}+4 c_{j}+c_{j+1}, \\
U_{j}^{\prime}=\frac{3}{h}\left(c_{j+1}-c_{j-1}\right), \\
U_{j}^{\prime \prime}=\frac{6}{h^{2}}\left(c_{j-1}-2 c_{j}+c_{j+1}\right) .
\end{gathered}
$$

\section{Numerical Scheme}

We have used the following modified form of cubic B-spline basis functions [21] in the combination with collocation, 
TABLE 2: $L_{2}$ and $L_{\infty}$ errors of Example 1 in the domain $[-1,1]$ at different time levels.

\begin{tabular}{lcccccc}
\hline & & \multicolumn{2}{c}{ Present Scheme } & \multicolumn{2}{c}{ Dehghan and Shokri [13] } \\
$t$ & $L_{2}$ & $h=.04$ & $L_{2}$ & $L_{\infty}$ & $L_{2}$ & $L_{\infty}$ \\
\hline .25 & $1.18 \times 10^{-5}$ & $2.32 \times 10^{-5}$ & $3.71 \times 10^{-6}$ & $8.20 \times 10^{-6}$ & $3.91 \times 10^{-5}$ & $5.89 \times 10^{-6}$ \\
.50 & $4.19 \times 10^{-5}$ & $4.11 \times 10^{-5}$ & $1.34 \times 10^{-5}$ & $1.62 \times 10^{-5}$ & $1.30 \times 10^{-4}$ & $2.01 \times 10^{-5}$ \\
.75 & $7.78 \times 10^{-5}$ & $1.02 \times 10^{-4}$ & $2.40 \times 10^{-5}$ & $2.54 \times 10^{-5}$ & $2.35 \times 10^{-4}$ & $3.63 \times 10^{-5}$ \\
1 & $1.30 \times 10^{-4}$ & $1.64 \times 10^{-4}$ & $3.00 \times 10^{-5}$ & $4.14 \times 10^{-5}$ & $3.27 \times 10^{-4}$ & $5.07 \times 10^{-5}$ \\
\hline
\end{tabular}

TABLE 3: Absolute errors of Example 1 at different time levels with $h=.02, \Delta t=.0001$.

\begin{tabular}{lccc}
\hline$x$ & $t=.01$ & $t=.1$ & $t=1$ \\
\hline-.80 & $4.19 \times 10^{-11}$ & $4.24 \times 10^{-8}$ & $1.11 \times 10^{-5}$ \\
-.60 & $1.72 \times 10^{-11}$ & $1.94 \times 10^{-8}$ & $6.17 \times 10^{-7}$ \\
-.40 & $3.32 \times 10^{-11}$ & $3.01 \times 10^{-8}$ & $1.47 \times 10^{-5}$ \\
0 & $1.15 \times 10^{-10}$ & $1.09 \times 10^{-7}$ & $4.13 \times 10^{-5}$ \\
.40 & $3.32 \times 10^{-11}$ & $3.01 \times 10^{-8}$ & $1.47 \times 10^{-5}$ \\
.60 & $1.72 \times 10^{-11}$ & $1.94 \times 10^{-8}$ & $6.17 \times 10^{-7}$ \\
.80 & $4.19 \times 10^{-11}$ & $4.24 \times 10^{-8}$ & $1.11 \times 10^{-5}$ \\
\hline
\end{tabular}

to solve the sine-Gordon equation. Modified cubic B-spline basis functions have been used for handling the Dirichlet boundary conditions and finally we obtain a diagonally dominant system of differential equations. The procedure for modifying the basis functions is given as follows:

$$
\begin{aligned}
\widetilde{B}_{0}(x) & =B_{0}(x)+2 B_{-1}(x), \\
\widetilde{B}_{1}(x) & =B_{1}(x)-B_{-1}(x), \\
\widetilde{B}_{j}(x) & =B_{j}(x), \quad \text { for } j=2, \ldots, N-2, \\
\widetilde{B}_{N-1}(x) & =B_{N-1}(x)-B_{N+1}(x), \\
\widetilde{B}_{N}(x) & =B_{N}(x)+2 B_{N+1}(x) .
\end{aligned}
$$

To find the numerical solution of sine-Gordon equation (1), first it is rewritten as a pair of coupled equations using the following transformation:

$$
u_{t}(x, t)=v(x, t) .
$$

Then (1) transforms into a coupled system of equations as

$$
\begin{gathered}
u_{t}=v, \\
v_{t}=u_{x x}-\sin u .
\end{gathered}
$$

Now for solving the couple of (10), using collocation method with modified cubic B-spline basis functions, first we assume our solution as the linear combination of modified cubic B-spline basis functions:

$$
U_{N}(x, t)=\sum_{j=0}^{N} c_{j}(t) \widetilde{B}_{j}(x) .
$$

Using the approximate solution (11), the approximate value of $U_{t}(x)$ can be written as

$$
U_{t}(x)=\sum_{j=0}^{N} \dot{c}_{j}(t) \widetilde{B}_{j}(x),
$$

where $\dot{c}_{j}(t)$ is the derivative of $c_{j}(t)$ with respect to time $t$.

Using modified basis function (8) and Table 1 in (12), the value of $U_{t}(x)$ at different knots can be written as

$$
\begin{aligned}
U_{t}\left(x_{0}\right)=6 \dot{c}_{0}, & j=0, \\
U_{t}\left(x_{j}\right)=\dot{c}_{j-1}+4 \dot{c}_{j}+\dot{c}_{j+1}, & j=1,2, \ldots, N-1, \\
U_{t}\left(x_{N}\right)=6 \dot{c}_{N}, & j=N .
\end{aligned}
$$

Using (11) in coupled system (10) and imposing the boundary conditions (3) at the boundary points, we have

$$
\begin{gathered}
U_{t}\left(x_{0}\right)=\dot{\psi}_{1}(t), \quad j=0, \\
U_{t}\left(x_{j}\right)=v_{j}, \quad j=1,2, \ldots, N-1, \\
U_{t}\left(x_{N}\right)=\dot{\psi}_{2}(t), \quad j=N, \\
v_{t}\left(x_{0}\right)=\dot{g}_{1}(t), \quad \text { for } j=0, \\
v_{t}\left(x_{j}\right)=\sum_{j=0}^{N} c_{j} \widetilde{B}_{j}^{\prime \prime}(x)-\sin \left(U_{j}\right), \quad \text { for } j=1,2, \ldots, N-1, \\
v_{t}\left(x_{N}\right)=\dot{g}_{2}(t), \quad \text { for } j=N,
\end{gathered}
$$

where $g_{1}(t)$ and $g_{2}(t)$ are $\dot{\psi}_{1}(t)$ and $\dot{\psi}_{2}(t)$, respectively.

Now using (13) in (14) and (8) and Table 1 in (15), we get the following system of equations:

$$
\begin{gathered}
6 \dot{c}_{0}=\dot{\psi}_{1}(t), \quad j=0, \\
\dot{c}_{j-1}+4 \dot{c}_{j}+4 \dot{c}_{j+1}=v_{j}, \quad j=1,2, \ldots, N-1, \\
6 \dot{c}_{N}=\dot{\psi}_{2}(t), \quad j=N, \\
\dot{v}_{0}=\dot{g}_{1}(t), \quad j=0, \\
\dot{v}_{j}=\frac{6}{h^{2}}\left(c_{j-1}-2 c_{j}+c_{j+1}\right)-\sin \left(c_{j-1}+4 c_{j}+c_{j+1}\right), \\
j=1,2, \ldots, N-1, \\
\dot{v}_{N}=\dot{g}_{2}(t), \quad j=N .
\end{gathered}
$$


TABLE 4: $L_{\infty}$ and RMS errors of Example 1 in the domain $[-2,2]$ with $h=.01, \Delta t=.01$ at different time levels.

\begin{tabular}{lcccc}
\hline$t$ & \multicolumn{2}{c}{ Present Scheme } & \multicolumn{2}{c}{ Li-Min and Zong-Min [16] } \\
\hline .1 & $L_{\infty}$ & RMS & $1.54 \times 10^{-6}$ & $7.43 \times 10^{-6}$ \\
.2 & $7.20 \times 10^{-6}$ & $6.53 \times 10^{-8}$ & $9.25 \times 10^{-5}$ & $1.76 \times 10^{-5}$ \\
.3 & $2.26 \times 10^{-5}$ & $2.69 \times 10^{-7}$ & $9.02 \times 10^{-5}$ & $3.60 \times 10^{-5}$ \\
.4 & $4.54 \times 10^{-5}$ & $6.36 \times 10^{-7}$ & $1.62 \times 10^{-4}$ & $1.62 \times 10^{-4}$ \\
.5 & $7.52 \times 10^{-5}$ & $1.19 \times 10^{-6}$ & $2.58 \times 10^{-4}$ & $1.10 \times 10^{-4}$ \\
.6 & $1.12 \times 10^{-4}$ & $1.96 \times 10^{-6}$ & $3.73 \times 10^{-4}$ & $1.65 \times 10^{-4}$ \\
.7 & $1.55 \times 10^{-4}$ & $2.96 \times 10^{-6}$ & $4.98 \times 10^{-4}$ & $2.29 \times 10^{-4}$ \\
.8 & $2.04 \times 10^{-4}$ & $4.21 \times 10^{-6}$ & $6.24 \times 10^{-4}$ & $2.98 \times 10^{-4}$ \\
.9 & $2.59 \times 10^{-4}$ & $5.72 \times 10^{-6}$ & $7.44 \times 10^{-4}$ & $3.69 \times 10^{-4}$ \\
1 & $3.19 \times 10^{-4}$ & $7.50 \times 10^{-6}$ & $8.49 \times 10^{-4}$ & $4.37 \times 10^{-4}$ \\
\hline
\end{tabular}

TABLE 5: $L_{2}$ and $L_{\infty}$ errors of Example 2 with $c=.5$ at different time levels.

\begin{tabular}{|c|c|c|c|c|c|c|}
\hline \multirow{3}{*}{$t$} & \multicolumn{4}{|c|}{ Present Scheme } & \multirow{2}{*}{\multicolumn{2}{|c|}{$\begin{array}{l}\text { Dehghan and Shokri [13] } \\
\qquad h=.04\end{array}$}} \\
\hline & \multicolumn{2}{|c|}{$h=.04$} & \multicolumn{2}{|c|}{$h=.02$} & & \\
\hline & $L_{2}$ & $L_{\infty}$ & $L_{2}$ & $L_{\infty}$ & $L_{2}$ & $L_{\infty}$ \\
\hline .25 & $3.66 \times 10^{-5}$ & $4.90 \times 10^{-5}$ & $9.26 \times 10^{-6}$ & $1.21 \times 10^{-6}$ & $1.76 \times 10^{-5}$ & $4.95 \times 10^{-6}$ \\
\hline .50 & $9.00 \times 10^{-5}$ & $7.55 \times 10^{-5}$ & $2.24 \times 10^{-5}$ & $1.89 \times 10^{-5}$ & $4.31 \times 10^{-5}$ & $8.42 \times 10^{-6}$ \\
\hline .75 & $1.60 \times 10^{-4}$ & $1.43 \times 10^{-4}$ & $3.98 \times 10^{-5}$ & $3.57 \times 10^{-5}$ & $8.25 \times 10^{-5}$ & $1.65 \times 10^{-5}$ \\
\hline 1 & $2.27 \times 10^{-4}$ & $2.10 \times 10^{-4}$ & $5.66 \times 10^{-5}$ & $5.25 \times 10^{-5}$ & $1.27 \times 10^{-4}$ & $2.51 \times 10^{-5}$ \\
\hline
\end{tabular}

TABLE 6: Absolute errors of Example 2 at different time levels with $h=.02, \Delta t=.0001$.

\begin{tabular}{lccc}
\hline$x$ & $t=.01$ & $t=.1$ & $t=1$ \\
\hline-2.5 & $6.05 \times 10^{-10}$ & $5.96 \times 10^{-8}$ & $5.28 \times 10^{-6}$ \\
-2 & $8.76 \times 10^{-10}$ & $8.69 \times 10^{-8}$ & $1.21 \times 10^{-6}$ \\
-1.5 & $5.64 \times 10^{-10}$ & $5.89 \times 10^{-8}$ & $9.16 \times 10^{-8}$ \\
-1 & $2.68 \times 10^{-9}$ & $2.53 \times 10^{-7}$ & $2.02 \times 10^{-5}$ \\
0 & $5.80 \times 10^{-11}$ & $5.64 \times 10^{-8}$ & $2.51 \times 10^{-5}$ \\
1 & $2.72 \times 10^{-9}$ & $2.92 \times 10^{-7}$ & $4.82 \times 10^{-5}$ \\
1.5 & $5.57 \times 10^{-10}$ & $5.08 \times 10^{-8}$ & $1.27 \times 10^{-5}$ \\
2 & $8.78 \times 10^{-10}$ & $8.81 \times 10^{-8}$ & $2.21 \times 10^{-6}$ \\
2.5 & $6.07 \times 10^{-10}$ & $6.15 \times 10^{-8}$ & $3.41 \times 10^{-6}$ \\
\hline
\end{tabular}

The systems (16) represent a system of first order differential equations and can be written as

$$
\begin{gathered}
{\left[\begin{array}{ccccc}
6 & 0 & \cdots & \cdots & 0 \\
1 & 4 & 1 & \cdots & 0 \\
& & \cdots & \cdots & \cdots \\
& & \cdots & \cdots & \cdots \\
& & 1 & 4 & 1 \\
& & 0 & 6
\end{array}\right]\left[\begin{array}{c}
\dot{c}_{0} \\
\dot{c}_{1} \\
\cdots \\
\cdots \\
\dot{c}_{N-1} \\
\dot{c}_{N}
\end{array}\right]=\left[\begin{array}{c}
F_{0} \\
F_{1} \\
\cdots \\
\cdots \\
F_{N-1} \\
F_{N}
\end{array}\right],} \\
{\left[\begin{array}{c}
\dot{v}_{0} \\
\dot{v}_{1} \\
\cdots \\
\cdots \\
\dot{v}_{N-1} \\
\dot{v}_{N}
\end{array}\right]}
\end{gathered}
$$

where

$$
\begin{gathered}
F_{0}=\dot{\psi}_{1}(t), \quad G_{0}=\dot{g}_{1}(t), \quad F_{j}=v_{j}, \\
G_{j}=\frac{6}{h^{2}}\left(c_{j-1}-2 c_{j}+c_{j+1}\right)-\sin \left(c_{j-1}+4 c_{j}+c_{j+1}\right), \\
j=1,2, \ldots, N-1, \\
F_{N}=\dot{\psi}_{2}(t), \quad G_{N}=\dot{g}_{2}(t) .
\end{gathered}
$$

Once the vector $\mathbf{c}^{\mathbf{n}}$ has been determined at a specific time level, using (7), we can compute the approximate solution at the required knots. So first we solve system (17) for vector $\dot{\mathbf{c}}$, by using Thomas algorithm only once at each time level $t>0$. Then the obtained system with the system (18) will give $(2 \mathrm{~N}+$ 2) first order ordinary differential equations and finally $(2 \mathrm{~N}+$ 2) first order ordinary differential equations have been solved by SSP-RK54 [20] scheme and consequently the approximate solution $U_{N}(x, t)$ is computed.

\section{Computation of Initial Vector}

To find the solution at specific time level $t>0$, we need the initial vectors $\mathbf{c}^{\mathbf{0}}$ and $\mathbf{v}^{\mathbf{0}}$.

Using initial conditions (2), we have the following.

4.1. Initial Vector $\mathbf{c}^{0}$. Consider the following:

$$
\begin{gathered}
U\left(x_{j}, 0\right)=\psi_{1}(0), \quad j=0, \\
U\left(x_{j}, 0\right)=\phi_{1}\left(x_{j}\right), \quad j=1, \ldots, N-1, \\
U\left(x_{j}, 0\right)=\psi_{2}(0), \quad j=N .
\end{gathered}
$$


TABLE 7: Errors and order of convergence for Example 2 at $t=1$.

\begin{tabular}{lcccc}
\hline$h$ & $L_{2}$ & Order of convergence & $L_{\infty}$ & Order of convergence \\
\hline .08 & $9.08 \times 10^{-4}$ & & $8.40 \times 10^{-4}$ & 2 \\
.04 & $2.27 \times 10^{-4}$ & 2 & $2.10 \times 10^{-4}$ & 2 \\
.02 & $5.66 \times 10^{-5}$ & 2.003 & $5.25 \times 10^{-5}$ & 2.002 \\
.01 & $1.40 \times 10^{-5}$ & 2.015 & $1.31 \times 10^{-5}$ & 1.998 \\
.005 & $3.65 \times 10^{-6}$ & 1.939 & $3.28 \times 10^{-6}$ & \\
\hline
\end{tabular}

TABLE 8: $L_{2}$ and $L_{\infty}$ errors of Example 3 with $c=.5, h=.01, \Delta t=.001$ at different time levels.

\begin{tabular}{lcccc}
\hline$t$ & \multicolumn{2}{c}{ Present Scheme } & Bratsos [12] & Uddin et al. [19] \\
\hline 1 & $L_{2}$ & $L_{\infty}$ & $L_{\infty}$ & $L_{\infty}$ \\
10 & $7.448 \times 10^{-6}$ & $7.029 \times 10^{-6}$ & $.98816 \times 10^{-3}$ & $1.474 \times 10^{-3}$ \\
20 & $3.999 \times 10^{-5}$ & $2.226 \times 10^{-5}$ & $.16291 \times 10^{-2}$ & $9.215 \times 10^{-3}$ \\
\hline
\end{tabular}

System $(20)$ is a $(N+1) \times(N+1)$ tridiagonal system of equations, which can be written as

$$
A \mathbf{c}^{0}=B
$$

where

$$
\begin{gathered}
A=\left[\begin{array}{ccccc}
6 & 0 & \cdots & \cdots & 0 \\
1 & 4 & 1 & \cdots & 0 \\
& & \cdots & \cdots & \cdots \\
& & \cdots & \cdots & \cdots \\
& 1 & 4 & 1 \\
& & 0 & 6
\end{array}\right], \quad \mathbf{c}^{\mathbf{0}}=\left[\begin{array}{c}
\dot{c}_{0} \\
\dot{c}_{1} \\
\cdots \\
\cdots \\
\dot{c}_{N-1} \\
\dot{c}_{N}
\end{array}\right], \\
B=\left[\begin{array}{c}
\psi_{1}(0) \\
\phi_{1}\left(x_{1}\right) \\
\cdots \\
\cdots \\
\phi_{1}\left(x_{N-1}\right) \\
\psi_{2}(0)
\end{array}\right] .
\end{gathered}
$$

$A$ is tridiagonal matrix. Using Thomas algorithm the solution of (20) can be easily found.

4.2. Initial Vector $\mathbf{v}^{\mathbf{0}}$. Using the initial condition

$$
U_{t}(x, 0)=\phi_{2}(x)
$$

we have

$$
v\left(x_{j}, 0\right)=\phi_{2}\left(x_{j}\right), \quad j=0,1, \ldots, N-1, N,
$$

which gives the initial vector $\mathbf{v}^{\mathbf{0}}$.

\section{Numerical Experiments}

In this section, we consider four numerical examples to validate the proposed scheme. The accuracy of the scheme is verified by calculating $L_{2}, L_{\infty}$, and root mean square errors and results are also compared with some published work.
$L_{2}, L_{\infty}$, and RMS error norms are given by the following formulae:

$$
\begin{aligned}
& L_{2}=\left\|u-U_{N}\right\|_{2}=\sqrt{h \sum_{j=0}^{N}\left|u_{j}-\left(U_{N}\right)_{j}\right|^{2}}, \\
& L_{\infty}=\left\|u-U_{N}\right\|_{\infty}=\max _{j}\left|u_{j}-\left(U_{N}\right)_{j}\right|
\end{aligned}
$$

Example 1. In this example the numerical solutions of (1) are obtained in the computational domain $x \in[-a, a]$ with the initial conditions

$$
u(x, 0)=0, \quad u_{t}(x, 0)=4 \operatorname{sech}(x)
$$

The exact solution $[6,15,16]$ is given as

$$
u(x, t)=4 \arctan (t \operatorname{sech}(x)) .
$$

The boundary conditions (3) are obtained from the exact solution.

Case I (when $a=1$ ). First we solve the above example in the computational domain $[-1,1]$ with $\Delta t=.0001$ and for space step sizes $h=.02, .04$. In Table 2 , we report the $L_{2}$ and $L_{\infty}$ errors and compare them with those given in Dehghan and Shokri [13]. We see that our results are in good agreement with [13], when we take $h=.04$. For $h=.02$ our results are better than the result of [13] in terms of $L_{2}$ error. In Table 3, absolute errors, are reported at $t=.01, .1,1$, with $h=.02$. A graph comparing the exact and numerical solutions at $t=1$ with $h=.02$ and $\Delta t=.0001$ is depicted in Figure 1 .

Case II. In the domain $[-2,2]$, the solutions of Example 1 are obtained with $\Delta t=.01$ and $h=.01$. In Table 4, we report the $L_{\infty}$ and RMS errors at different time levels and results are compared with those of Li-Min and Zong-Min [16]. We 
TABLE 9: Errors and order of convergence for Example 3 at $t=20$.

\begin{tabular}{lcccc}
\hline$h$ & $L_{2}$ & Order of convergence & $L_{\infty}$ & Order of convergence \\
\hline .08 & $4.171 \times 10^{-2}$ & & $2.302 \times 10^{-2}$ & \\
.04 & $1.038 \times 10^{-2}$ & 2.006 & $5.729 \times 10^{-3}$ & 2.006 \\
.02 & $2.593 \times 10^{-3}$ & 2.001 & $1.430 \times 10^{-3}$ & 2.002 \\
.01 & $6.467 \times 10^{-4}$ & 2.003 & $3.567 \times 10^{-4}$ & 2.003 \\
\hline
\end{tabular}

TABLE 10: $L_{2}$ and $L_{\infty}$ errors of Example 4 with $c=.5$ at different time levels.

\begin{tabular}{|c|c|c|c|c|}
\hline \multirow{3}{*}{$t$} & \multirow{2}{*}{\multicolumn{2}{|c|}{$\begin{array}{c}\text { Present Scheme } \\
\quad h=.01\end{array}$}} & \multirow{3}{*}{$\begin{array}{c}\text { Bratsos }[12] \\
h=.01 \\
L_{\infty}\end{array}$} & \multirow{3}{*}{$\begin{array}{c}\text { Uddin et al. [19] } \\
\qquad N=201 \\
L_{\infty}\end{array}$} \\
\hline & & & & \\
\hline & $L_{2}$ & $L_{\infty}$ & & \\
\hline 2 & $2.564 \times 10^{-5}$ & $1.818 \times 10^{-5}$ & $.12760 \times 10^{-3}$ & $1.568 \times 10^{-3}$ \\
\hline 10 & $8.850 \times 10^{-5}$ & $5.228 \times 10^{-5}$ & $.19115 \times 10^{-3}$ & $3.151 \times 10^{-3}$ \\
\hline 20 & $1.713 \times 10^{-4}$ & $9.438 \times 10^{-5}$ & $.25189 \times 10^{-3}$ & $1.828 \times 10^{-2}$ \\
\hline
\end{tabular}

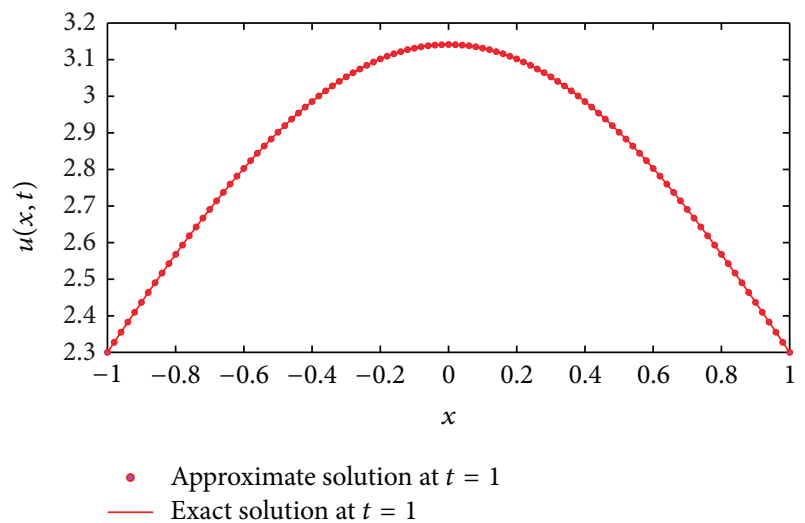

FIgURE 1: Approximate and exact solution at $t=1$ with $h=.02$ and $\Delta t=.0001$.

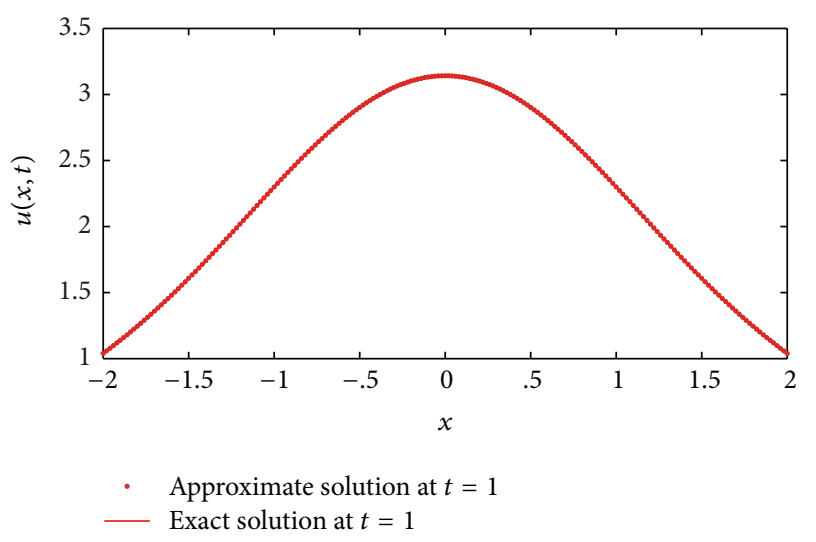

Figure 2: Approximate and exact solution at $t=1$ with $h=.01$ and $\Delta t=.01$.

noticed that our results are in good agreement with [16] in terms of $L_{\infty}$ error and in terms of RMS error our results are better than [16]. Figure 2 depicts the comparison of exact and numerical solution at $t=1$.
Example 2. In this example we consider (1) in the computational domain $x \in[-3,3]$ with the initial conditions

$$
\begin{gathered}
u(x, 0)=4 \arctan \left(\exp \left(\frac{x}{\sqrt{1-c^{2}}}\right)\right), \\
u_{t}(x, 0)=\frac{-4 c \gamma \exp (\gamma x)}{1+\exp (2 \gamma x)}
\end{gathered}
$$

and the exact solution $[6,15]$ is given by

$$
u(x, t)=4 \arctan (\exp (\gamma(x-c t))),
$$

where $c$ is the velocity of solitary wave and $\gamma=1 / \sqrt{1-c^{2}}$.

The boundary conditions (3) can be obtained from the exact solution.

In Table 5, we report $L_{2}$ and $L_{\infty}$ errors, for different values of $h$ with $c=.5$ and $\Delta t=.0001$. We also compare our results with those of Dehghan and Shokri [13]. From Table 5, it is clear that our scheme and that of [13] have approximately similar $L_{2}$ errors, for $h=.04$. For $h=.02$ our results are better than [13] in terms of $L_{2}$ errors and approximately similar in terms of $L_{\infty}$ error norm. We also compute $L_{2}, L_{\infty}$ error norm for different values of $h$ with $\Delta t=.0001$ at $t=1$ and calculate order of convergence, which is shown in Table 7 . The absolute errors, for $t=.01, .1,1$, are also reported in Table 6 . Figure 3 depicts the graph between exact and numerical solutions at $t=1$.

Example 3. In this example we consider nonlinear sineGordon equation in the computational domain $x \in[-10,10]$ with the initial conditions

$$
u(x, 0)=0, \quad u_{t}(x, 0)=\frac{4}{\sqrt{1+c^{2}}} \operatorname{sech}\left(\frac{x}{\sqrt{1+c^{2}}}\right) .
$$

The exact solution $[12,19]$ is given as

$$
u(x, t)=4 \arctan \left(c^{-1} \sin (\bar{\gamma} c t) \operatorname{sech}(\bar{\gamma} x)\right),
$$

where $c$ is the velocity of solitary wave and $\bar{\gamma}=1 / \sqrt{1+c^{2}}$. 
TABLE 11: Errors and order of convergence for Example 4 at $t=20$.

\begin{tabular}{lcccc}
\hline$h$ & $L_{2}$ & Order of convergence & $L_{\infty}$ & Order of convergence \\
\hline .08 & $1.094 \times 10^{-2}$ & & $6.025 \times 10^{-3}$ & \\
.04 & $2.740 \times 10^{-3}$ & 1.997 & $1.509 \times 10^{-3}$ & 1.997 \\
.02 & $6.851 \times 10^{-4}$ & 1.999 & $3.775 \times 10^{-4}$ & 1.999 \\
.01 & $1.713 \times 10^{-4}$ & 1.999 & $9.438 \times 10^{-5}$ & 1.999 \\
\hline
\end{tabular}

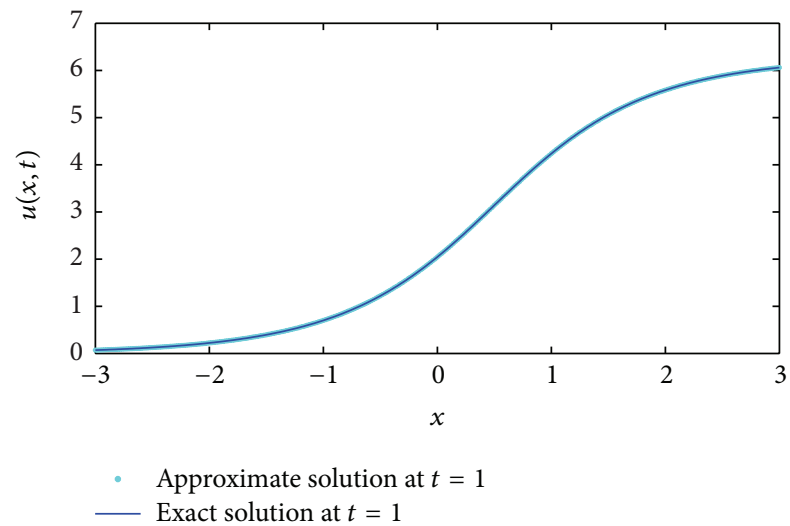

Figure 3: Approximate and exact solution at $t=1$ with $h=.02$ and $\Delta t=.0001$.

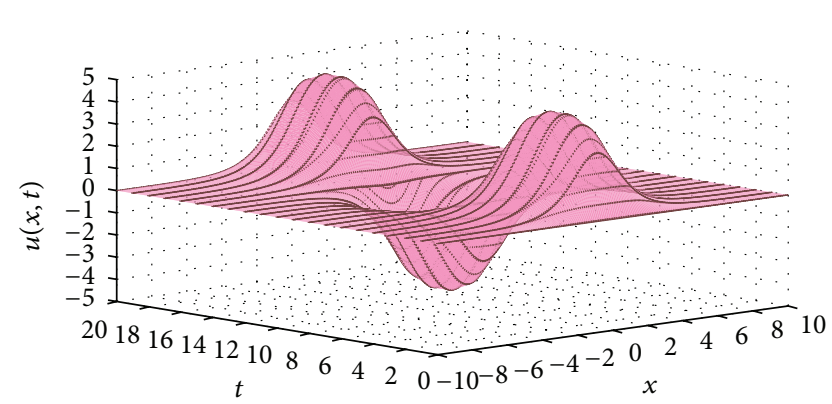

FIGURE 4: The graph of approximate solution up to $t=20$, in domain $-10 \leq x \leq 10$.

The boundary conditions (3) can be obtained from the exact solution.

We solve Example 3 with $c=.5, h=.01$, and $\Delta t=$ .001. Computed $L_{2}$ and $L_{\infty}$ errors are reported in Table 8 and compared with Uddin et al. [19] and Bratsos [12]. As we see from Table 8 , our results are better. In Table 9, we report $L_{2}$ and $L_{\infty}$ error norms for different values of $h$ with $\Delta t=.001$ at $t=20$, and using them we compute the order of convergence. It is clear that the present method is of second order of convergence. In Figure 4, we show the space-time graph of approximate solution for $t \leq 20$ with $h=.01$ and $\Delta t=.001$.

Example 4. Now we consider (1) in the computational domain $x \in[-20,20]$ with the initial conditions

$$
u(x, 0)=4 \arctan \left(c \sinh \left(\frac{x}{\sqrt{1-c^{2}}}\right)\right), \quad u_{t}(x, 0)=0 .
$$

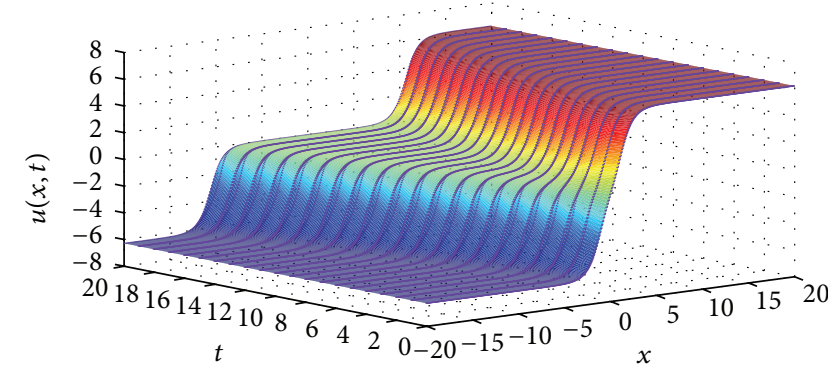

FIGURE 5: The graph of approximate solution up to $t=20$, in domain $-20 \leq x \leq 20$.

The exact solution $[12,19]$ is given as

$$
u(x, t)=4 \arctan (c \sinh (\gamma x) \operatorname{sech}(\gamma c t)),
$$

where $c$ is the velocity of solitary wave and $\gamma=1 / \sqrt{1-c^{2}}$. The boundary conditions (3) can be obtained from the exact solution.

We solve Example 4 with $c=.5, h=.01$, and $\Delta t=.001$ and results are reported in Table 10 . We compare our results with those given in Uddin et al. [19] and Bratsos [12] in terms of $L_{\infty}$ errors and found them better. In Table 11, we report the $L_{2}$ and $L_{\infty}$ error norms for different values of $h$, with $\Delta t=.001$ at $t=20$, and using them compute the order of convergence of the method. Figure 5 depicts the space-time graph of approximate solution for $t \leq 20$ with $h=.01$ and $\Delta t=.001$.

\section{Conclusions}

This paper investigates the application of B-spline collocation method to represent the solution of sine-Gordon equation with Dirichlet's boundary conditions, by reducing it into coupled system of equations. The combination of modified cubic B-spline basis function in space and SSP-RK54 in time successfully worked to give reliable and accurate solutions of sine-Gordon equation. The numerical approximate solutions have been computed without using any transformation and linearization process. To demonstrate the accuracy and usefulness of the present scheme, four numerical experiments are carried out and results are compared with some earlier work. It is observed that the proposed method produces good results in comparison to those available in the literature. From the order of convergence calculated in Tables 7, 9, and 11, the method is shown to have a second order of convergence. 
The results reveal that the present method is an applicable technique and approximates the solutions very well.

\section{Conflict of Interests}

The authors declare that there is no conflict of interests regarding the publication of this paper.

\section{References}

[1] J. K. Perring and T. H. R. Skyrme, "A model unified field equation," Nuclear Physics, vol. 31, pp. 550-555, 1962.

[2] A. Barone, F. Esposito, C. J. Magee, and A. C. Scott, "Theory and applications of the sine-Gordon equation," La Rivista del Nuovo Cimento, vol. 1, no. 2, pp. 227-267, 1971.

[3] M. Dehghan and A. Shokri, "Numerical solution of the nonlinear Klein-Gordon equation using radial basis functions," Journal of Computational and Applied Mathematics, vol. 230, no. 2, pp. 400-410, 2009.

[4] G. Ben-Yu, P. J. Pascual, M. J. Rodriguez, and L. Vázquez, "Numerical solution of the sine-Gordon equation," Applied Mathematics and Computation, vol. 18, no. 1, pp. 1-14, 1986.

[5] A. G. Bratsos and E. H. Twizell, "The solution of the sineGordon equation using the method of lines," International Journal of Computer Mathematics, vol. 61, no. 3-4, pp. 271-292, 1996.

[6] A. Mohebbi and M. Dehghan, "High-order solution of onedimensional sine-Gordon equation using compact finite difference and DIRKN methods," Mathematical and Computer Modelling, vol. 51, no. 5-6, pp. 537-549, 2010.

[7] J. X. Kuang and L. H. Lu, "Two classes of finite-difference methods for generalized sine-Gordon equations," Journal of Computational and Applied Mathematics, vol. 31, no. 3, pp. 389396, 1990.

[8] A. G. Bratsos and E. H. Twizell, "A family of parametric finite-difference methods for the solution of the sine-Gordon equation," Applied Mathematics and Computation, vol. 93, no. 2-3, pp. 117-137, 1998.

[9] G. W. Wei, "Discrete singular convolution for the sine-Gordon equation," Physica D, vol. 137, no. 3-4, pp. 247-259, 2000.

[10] B. Batiha, M. S. M. Noorani, and I. Hashim, "Numerical solution of sine-Gordon equation by variational iteration method," Physics Letters A, vol. 370, no. 5-6, pp. 437-440, 2007.

[11] C. Zheng, "Numerical solution to the sine-Gordon equation defined on the whole real axis," SIAM Journal on Scientific Computing, vol. 29, no. 6, pp. 2494-2506, 2007.

[12] A. G. Bratsos, "A fourth order numerical scheme for the onedimensional sine-Gordon equation," International Journal of Computer Mathematics, vol. 85, no. 7, pp. 1083-1095, 2008.

[13] M. Dehghan and A. Shokri, "A numerical method for onedimensional nonlinear sine-Gordon equation using collocation and radial basis functions," Numerical Methods for Partial Differential Equations, vol. 24, no. 2, pp. 687-698, 2008.

[14] M. Dehghan and D. Mirzaei, "The boundary integral equation approach for numerical solution of the one-dimensional sineGordon equation," Numerical Methods for Partial Differential Equations, vol. 24, no. 6, pp. 1405-1415, 2008.

[15] J. Rashidinia and R. Mohammadi, "Tension spline solution of nonlinear sine-Gordon equation," Numerical Algorithms, vol. 56, no. 1, pp. 129-142, 2011.
[16] M. Li-Min and W. Zong-Min, "A numerical method for onedimensional nonlinear sine-Gordon equation using multiquadric quasi-interpolation," Chinese Physics B, vol. 18, no. 8, pp. 3099-3103, 2009.

[17] Z.-W. Jiang and R.-H. Wang, "Numerical solution of onedimensional Sine-Gordon equation using high accuracy multiquadric quasi-interpolation," Applied Mathematics and Computation, vol. 218, no. 15, pp. 7711-7716, 2012.

[18] D. Kaya, "A numerical solution of the sine-Gordon equation using the modified decomposition method," Applied Mathematics and Computation, vol. 143, no. 2-3, pp. 309-317, 2003.

[19] M. Uddin, S. Haq, and G. Qasim, "A meshfree approach for the numerical solution of nonlinear sine-Gordon equation," International Mathematical Forum, vol. 7, no. 21-24, pp. 11791186, 2012.

[20] S. Gottlieb, C.-W. Shu, and E. Tadmor, "Strong stabilitypreserving high-order time discretization methods," SIAM Review, vol. 43, no. 1, pp. 89-112 (electronic), 2001.

[21] R. C. Mittal and R. Bhatia, "Numerical solution of second order one dimensional hyperbolic telegraph equation by cubic Bspline collocation method," Applied Mathematics and Computation, vol. 220, pp. 496-506, 2013. 


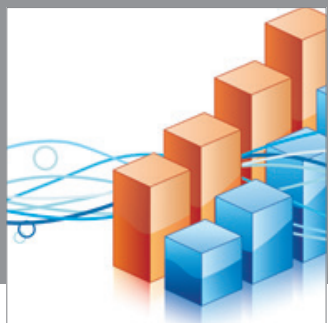

Advances in

Operations Research

mansans

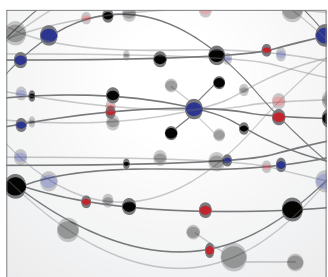

The Scientific World Journal
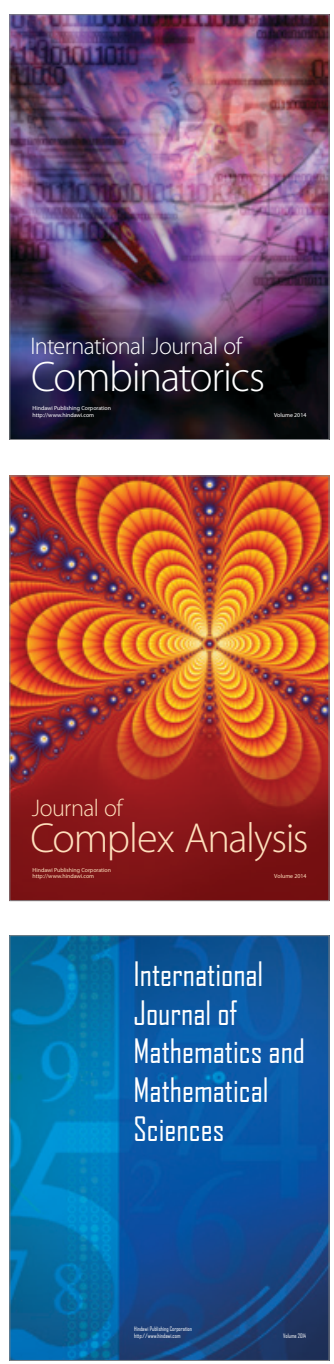
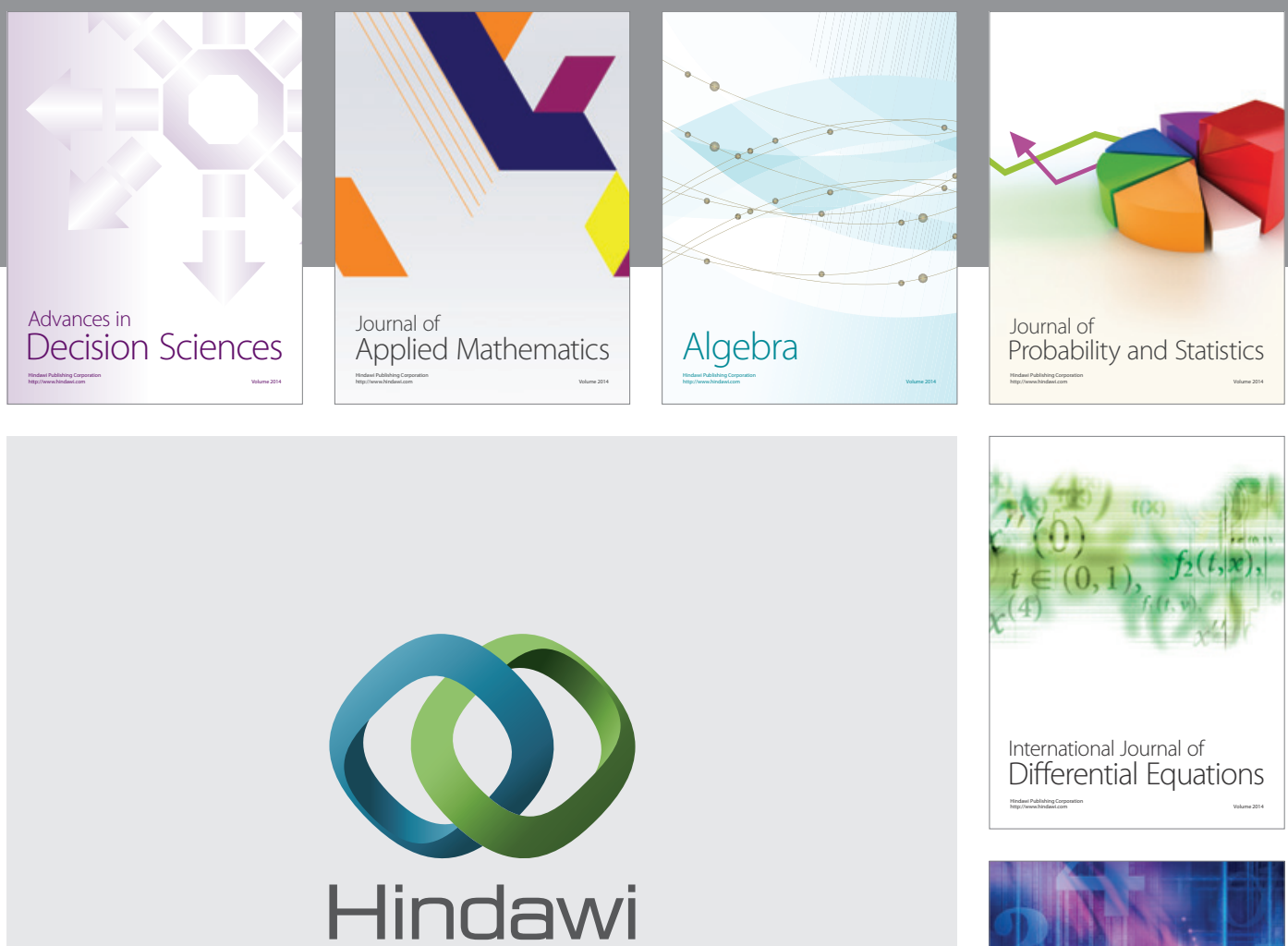

Submit your manuscripts at http://www.hindawi.com
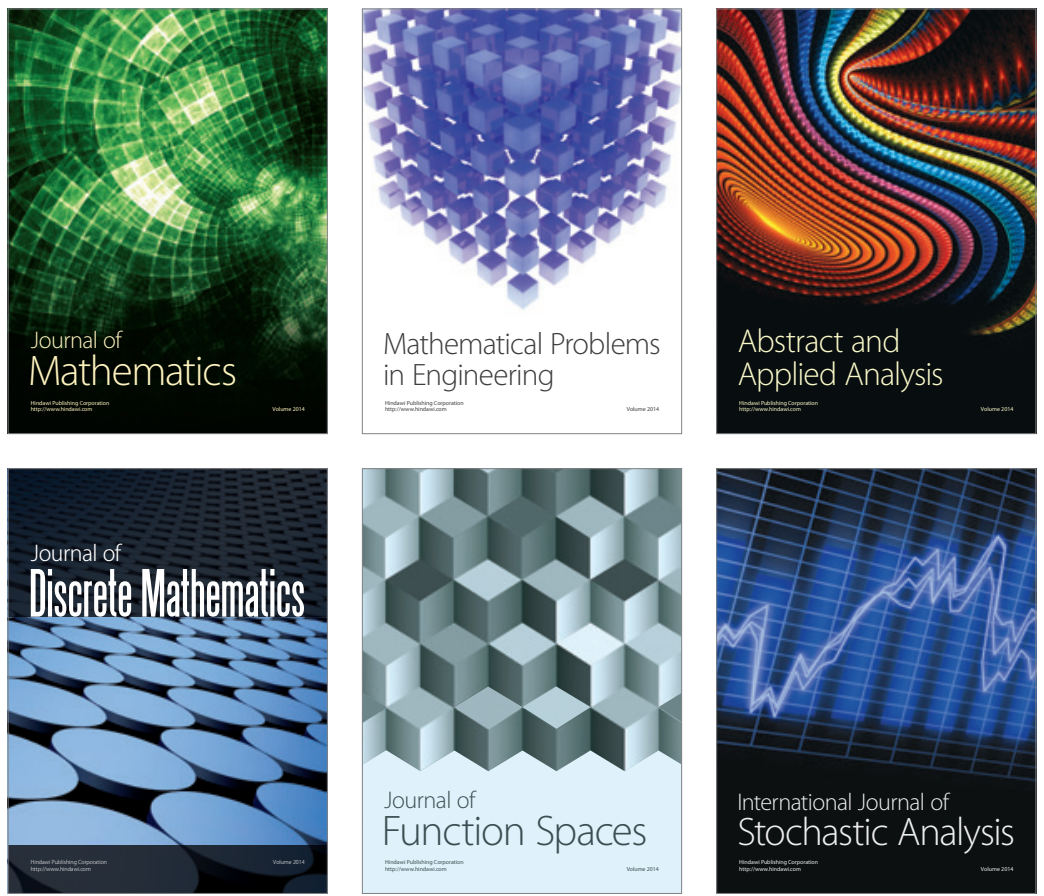

Journal of

Function Spaces

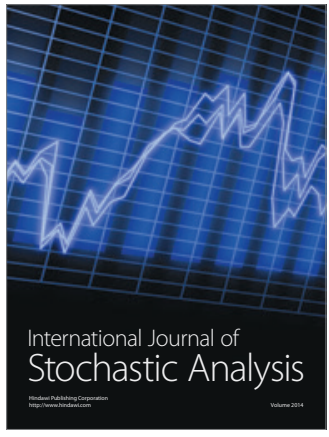

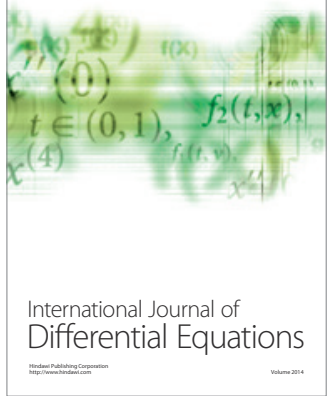
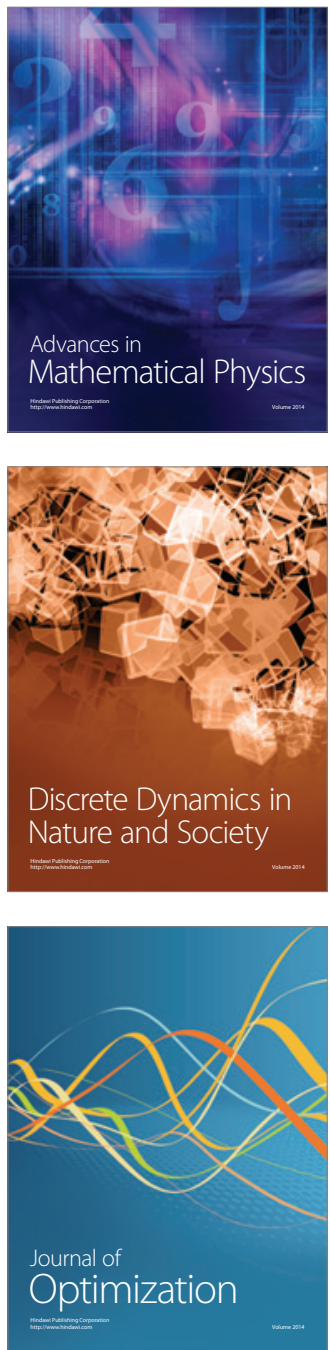